Europhys. Lett., 54 (6), pp. 729-735 (2001)

\title{
Vicinage effects in the charge state of swift molecular constituents traversing thin foils
}

\author{
S. Heredia-Avalos ${ }^{1}$, R. Garcia-Molina ${ }^{1}$ and N. R. Arista ${ }^{2}$ \\ 1 Departamento de Física, Universidad de Murcia \\ Apartado 4021, E-30080 Murcia, Spain \\ 2 Instituto Balseiro, Centro Atómico Bariloche \\ RA-8400 Bariloche, Argentina
}

(received 19 September 2000; accepted in final form 30 March 2001)

PACS. 34.50.Dy - Interactions of atoms and molecules with surfaces; photon and electron emission; neutralization of atoms.

PACS. 34.50.Fa - Electronic excitation and ionization of atoms (including beam-foil excitation and ionization).

PACS. 36.40.Wa - Charged clusters.

\begin{abstract}
We present a method to calculate the charge state of the atomic ions resulting from the fragmentation of swift molecular ions when traversing thin foils. The mutual influence of the neighbouring ions in the charge state of each atomic ion and the asymmetries in the screening close to the exit surface as each ion leaves the foil is taken into account. We have found the latter effect to be particularly important. Our calculations compare remarkably well with experimental data available for different molecular ions and foil thicknesses.
\end{abstract}

The charge state of a swift atomic ion moving through a solid is different when it is isolated or when it forms part of a cluster. In general, for a given velocity the average charge state of each molecular constituent is lower than that of the isolated atomic ion and depends on the molecular structure and velocity, as well as on the foil thickness [1-5]. This phenomenon is related to the proximity of the neighbouring atomic ions as they travel through the target, and it has been extensively studied, both theoretically and experimentally, during the last decades [1-7].

Recent experiments with $\mathrm{N}_{2}^{+}$and $\mathrm{C}_{n}^{+}(n=3-10)$ molecular ions incident on amorphous carbon foils $[3,5]$ show that the vicinage effects in the charge state of each molecular constituent decreases with the foil thickness and increases with the number of atomic ions that form the molecular ion.

In this work we present a model to calculate the charge state of an atomic ion, resulting from the fragmentation of a molecular ion, due to the vicinage effects produced by its neighbouring atomic ions, when they travel inside the solid target in a correlated manner.

When a swift molecular ion with velocity $v$ bombards a solid, it loses its binding electrons in the first atomic layers, and dissociates in its atomic constituents. Thereafter, mainly three processes affect the correlated motion of these atomic ions: the change in their electronic 
structures, the mutual Coulomb repulsion, and the energy loss due to the excitations produced in the stopping medium; all these processes are not independent, but mutually affected by the proximity of the atomic ions. For the energies and foil thicknesses to be discussed in this work, the energy loss is very small compared to the incident energy $[3,5]$, therefore we will only consider the first and second processes to analyse the vicinage effects in the charge state of each atomic ion as a function of the foil thickness and the molecular structure. In the following we explain and formulate the model and then we compare our calculations with the experimental data. Atomic units are used throughout this work, except where otherwise stated.

Let us consider a nuclear charge $Z$ surrounded by $N$ electrons, then the potential energy of these electrons interacting with the nucleus is

$$
U=-\sum_{i=1}^{N} \frac{Z}{r_{i}}
$$

where $r_{i}$ is the distance between the nucleus and the $i$-electron. Let us now consider an ensemble of $n$ atomic ions dissociated from a molecule. In the following we will center our study on one of these atomic ions, denoted by $k$. When this ion moves in correlation with the other ions, the potential energy of its $N_{k}^{*}$ electrons comes from the interaction with their own nucleus plus the interaction with the remaining $(n-1)$ atomic ions, namely

$$
U_{k}^{*}=-\sum_{i=1}^{N_{k}^{*}} \frac{1}{r_{k i}}\left[Z_{k}+\sum_{j \neq k}^{n}\left(Z_{j}-N_{j}^{*}\right) \frac{r_{k i}}{\left|\boldsymbol{R}_{k j}-\boldsymbol{r}_{k i}\right|} \exp \left[\frac{-\left|\boldsymbol{R}_{k j}-\boldsymbol{r}_{k i}\right|}{a}\right]\right] .
$$

To differentiate between a magnitude associated to an isolated or a correlated ion, an asterisk is added to the latter. Compared with eq. (1), the above expression has an extra term, which corresponds to the screened interaction of the $N_{k}^{*}$ electrons with the net charge $\left(Z_{j}-N_{j}^{*}\right)$ of each one of the $j \neq k$ neighbouring atomic ions. $\boldsymbol{R}_{k j}$ and $\boldsymbol{r}_{k i}$ are, respectively, the vectors from the $k$-nucleus to the $j$-nucleus and from the $k$-nucleus to its $i$-electron. The dynamic screening length $a$ is given by $a=\left(v^{2}+v_{\mathrm{F}}^{2} / 3\right)^{1 / 2} / \omega_{\mathrm{p}}$, which describes in a continuous manner the different behaviour for $v \geq v_{\mathrm{F}}$ and $v<v_{\mathrm{F}}[8] ; v_{\mathrm{F}}$ and $\omega_{\mathrm{p}}$ are the Fermi velocity and the plasmon frequency of the target. As compared to the isolated ion, the potential energy of the $N_{k}^{*}$ electrons is reduced by the proximity of the neighbouring ions, which will result in a stronger binding of these electrons.

It should be noted that the internal interaction between the electrons of a given ion is not included in eqs. (1) and (2) because these equations are used only to show the modification in the potential felt by a given electron due to the presence of external ions (with their corresponding electrons). We assume that the presence of other ions will not significantly affect the interelectronic interactions.

Depending on the internuclear separation, eq. (2) contains two limit cases. When $R_{k j} \rightarrow$ $\infty$, eq. (2) reproduces the potential energy for an isolated atomic ion, eq. (1). On the other hand, if $R_{k j}=0$, the $j$-nucleus is not screened by the target electrons with respect to the $k$-nucleus and $N_{j}^{*}=0$, then $a=\infty$ and the potential energy corresponds to the previously proposed united-atom model $[9,10]$.

It is not our purpose to calculate the fractions of each one of the possible charge states of the atomic ions. Therefore, in the following we will substitute $N_{j}^{*}$ and $N_{k}^{*}$ by the average values $\left\langle N_{j}^{*}\right\rangle$ and $\left\langle N_{k}^{*}\right\rangle$, respectively. Analogously, and considering the statistical character of the electron position, we will approximate $r_{k i}$ by a mean electronic radius $\left\langle r_{k}\right\rangle$, which we take 
from the Brandt-Kitagawa model [11] as follows:

$$
\left\langle r_{k}\right\rangle=\frac{0.96\left\langle N_{k}\right\rangle^{2 / 3}}{Z_{k}-\left\langle N_{k}\right\rangle / 7}
$$

where $\left\langle N_{k}\right\rangle$ is the average number of electrons bound to the isolated atomic ion. Equation (3) was deduced taking into account the interactions among all the electrons of a given ion $k$, which were not included in eqs. (1) and (2). It is important to note that the use of $\left\langle N_{k}\right\rangle$ instead of $\left\langle N_{k}^{*}\right\rangle$ will not modify significatively our calculation of the vicinage effects in the charge state. These previous considerations suggest that eq. (2) could be rewritten in a similar manner to eq. (1), but replacing the nuclear charge $Z_{k}$ by an effective nuclear charge $\mathcal{Z}_{k}$ that takes into account the reduction in energy due to the presence of the neighbouring atomic ions. Therefore,

$$
\mathcal{Z}_{k}=Z_{k}+\sum_{j \neq k}^{n}\left(Z_{j}-\left\langle N_{j}^{*}\right\rangle\right) \frac{\left\langle r_{k}\right\rangle}{R_{k j}} \exp \left[-\frac{R_{k j}}{a}\right] .
$$

To obtain the previous equation we have taken into account that the average of the potential felt by the $i$-electron due to the neighbour atomic ions is practically equal to the second term in the right-hand side of eq. (2) when $r_{k i} \ll R_{k j}$. Then, $\mathcal{Z}_{k}$ can be calculated provided the values of $\left\langle N_{j}^{*}\right\rangle$ are known, and these values can be statistically represented according to ref. [12] as follows:

$$
\left\langle N_{j}^{*}\right\rangle=\mathcal{Z}_{j} \exp \left[\frac{-0.92 v_{\mathrm{r}}}{\mathcal{Z}_{j}^{2 / 3}}\right],
$$

where $v_{\mathrm{r}}$ is the relative velocity of the $j$-atomic ion with respect to the valence electrons of the target [12],

$$
v_{\mathrm{r}}= \begin{cases}v\left(1+\frac{v_{\mathrm{F}}^{2}}{5 v^{2}}\right), & \text { when } v \geq v_{\mathrm{F}}, \\ \frac{3}{4} v_{\mathrm{F}}\left(1+\frac{2 v^{2}}{3 v_{\mathrm{F}}^{2}}-\frac{v^{4}}{15 v_{\mathrm{F}}^{4}}\right), & \text { when } v<v_{\mathrm{F}} .\end{cases}
$$

Equations (4) and (5) constitute a system of $2 n$ coupled non-linear equations, which can be solved numerically. This provides a self-consistent method to evaluate the average charge states of each one of the constituents of a molecular ion. As the values of $R_{k j}$ change with time due to the Coulomb repulsion, the value of the average charge state of each ion will also be a function of time (besides its dependence on the nature of the stopping medium and on the projectile velocity and geometry). Therefore, we have to consider the temporal evolution of the internuclear distances, $R_{k j}$, due to the Coulomb explosion [13] between the $n$ atomic ions with charge $Z_{j}-\left\langle N_{j}^{*}\right\rangle$.

To quantify the vicinage effects in the charge state we define the average charge state ratio as the quotient between the average charge state of the atomic ions within a cluster, and the average charge state of the same, but isolated, atomic ion,

$$
\mathcal{R}=\frac{\sum_{j=1}^{n}\left(Z_{j}-\left\langle N_{j}^{*}\right\rangle\right)}{\sum_{j=1}^{n}\left(Z_{j}-\left\langle N_{j}\right\rangle\right)} .
$$

It is important to note that the experimental charge state ratio, which is measured when the atomic ions exit the target, is different from the average charge state ratio inside the foil, because in the latter case the interaction between the atomic ions is screened by the electrons of the solid. 
To calculate the average charge state ratio suitable for comparison with experimental data, we must evaluate $\left\langle N_{j}^{*}\right\rangle$ in eq. (7) just after each ion exits the foil, because no further changes will take place while the ions travel in vacuum until reaching the detector. The $n$ atomic ions cross the surface of the foil in a sequential order, then we also have to consider their mutual interactions when some of them are outside and the others are inside the solid. To simplify the treatment, we assume a Heaviside step function for the surface electronic density, because its detailed form will not affect sensibly the final results [14]. The atomic ion that exits the foil in the $\ell$-th place acquires its average charge state under the influence of the remaining $(n-\ell)$ neighbours, screened by the electrons of the solid, plus the unscreened effect of the $(\ell-1)$ neighbours in the vacuum (i.e., with $a=\infty$ in eq. (4)). By combining the previous system of coupled equations (eqs. (4) and (5) for instantaneous internuclear distances $R_{k j}$ ) with the set of Newtonian equations determining the temporal evolution of $R_{k j}$ in terms of the instantaneous average charge states, we obtain a more general set of self-consistent equations that determine both the dynamics of the whole cluster and the temporal evolution of the average charge states.

Although the present treatment can be applied also to heteronuclear molecular ions, in the following we will use it for the case of the homonuclear $\mathrm{N}_{2}^{+}$and $\mathrm{C}_{n}^{+}(n=3-10)$ molecular ions incident on amorphous carbon foils $[3,5]$.

We will firstly analyse the case of $\mathrm{N}_{2}^{+}$ions moving through an amorphous carbon foil. To describe the temporal evolution of $R_{k j}$ it is necessary to specify the initial structure of the molecular ion, which, for the case of $\mathrm{N}_{2}^{+}$, is a linear molecule with internuclear distance 2.11 a.u. [15]. In this case, the system given by eqs. (4) and (5) is easy to solve. Figure 1 shows the average charge state ratio, eq. (7), for the constituent ions of $\mathrm{N}_{2}^{+}$as a function of the foil thickness, for a projectile energy of $2 \mathrm{MeV}$ /atom; the symbols correspond to experimental data [3] and the solid line is our calculation. The dashed line in fig. 1 shows the value of the average charge state ratio calculated before the atomic ions exit the foil. This figure clearly shows that the transient asymmetrical screening (due to the target electrons) at the exit of the foil is critical in order to explain properly the experimental data. In general, as fig. 1 shows, the charge state of the swift molecular constituents becomes smaller when emerging from the foil (larger vicinage effect) than it was inside it. This is because the screening inside the solid partially attenuates the vicinage effects in charge state.

Next we will study the case of $\mathrm{C}_{n}^{+}(n=3-10)$ ions traversing amorphous carbon foils [5], which will allow us to analyse the dependence of the vicinage effects in the charge state as a function of the molecular geometry and size. Figure 2(a) shows the average charge state ratio for a $\mathrm{C}_{3}^{+}$ion after traversing an amorphous carbon foil, as a function of the foil thickness, for a projectile energy of $2 \mathrm{MeV}$ /atom; the symbols show the experimental data [5]. Because the equilibrium structure for the $\mathrm{C}_{3}^{+}$ion is still in discussion [16-18], we have considered two possibilities: i) a linear structure or ii) an equilateral triangular structure, both with an internuclear distance of 2.4 a.u. [19]. The coupled system of eqs. (4) and (5) for a triangular $\mathrm{C}_{3}^{+}$ion is easy to solve, like the case of the $\mathrm{N}_{2}^{+}$ion. For linear $\mathrm{C}_{3}^{+}$ions we will use for simplicity $\left\langle N_{j}\right\rangle$, instead of $\left\langle N_{j}^{*}\right\rangle$, in eq. (4). The solid and dashed lines represent our calculations of $\mathcal{R}$ when we suppose a linear or a triangular structure for the $\mathrm{C}_{3}^{+}$ion, respectively. As fig. 2(a) clearly shows, the vicinage effects depend on the molecular geometry of the projectile. We can see that the calculations done for a linear structure are closer to the experimental data than the calculations done for the triangular structure (although the experimental error bars do not discard this structure completely). Therefore, a plausible conclusion is that the geometrical structure of the $\mathrm{C}_{3}^{+}$ion in the experimental conditions of ref. [5] could be linear. This fact is very interesting because the geometrical structure of the $\mathrm{C}_{3}^{+}$ion has been a subject of 


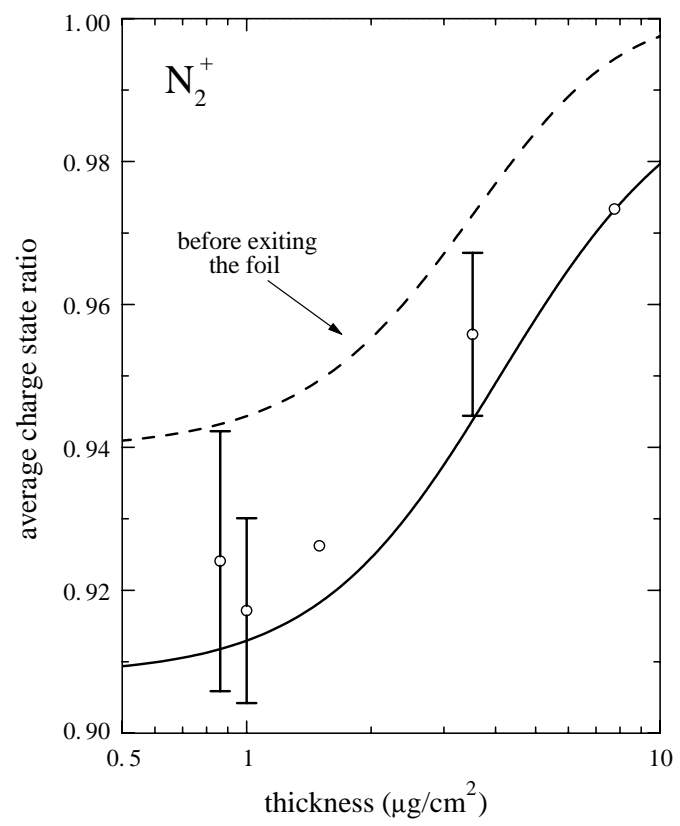

Fig. 1

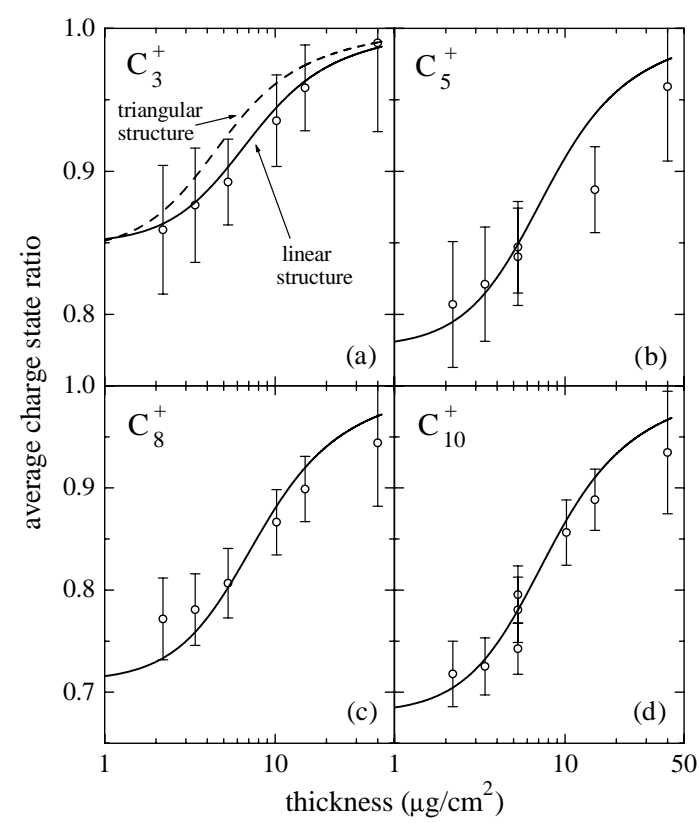

Fig. 2

Fig. 1 - Average charge state ratio, eq. (7), of the molecular constituents of $2 \mathrm{MeV} /$ atom $\mathrm{N}_{2}^{+}$incident on amorphous carbon, as a function of the foil thickness. The symbols show the experimental values [3] and the solid line is our calculation. The dashed line is the calculation considering the charge states before the atomic ions exit the foil.

Fig. 2 - Average charge state ratio, eq. (7), of the molecular constituents of $2 \mathrm{MeV} /$ atom $\mathrm{C}_{n}^{+}$ions incident on amorphous carbon, as a function of the foil thickness, for (a)- $n=3$, (b) $n=5$, (c) $n=8$, and (d) $n=10$. The symbols show the experimental values [5] and the solid lines represent our calculations for linear geometrical structures; the dashed line in panel (a) is our calculation for a triangular $\mathrm{C}_{3}^{+}$ion.

discussion for many years [16-18], and it seems that a detailed study of the charge state of the molecular constituents could help to elucidate this controversy.

Finally, we will consider a linear structure for the $\mathrm{C}_{n}^{+}(n=5,8,10)$ ions, with an internuclear distance of 2.4 a.u., according to ref. [19]. In figs. 2(b)-(d) we depict the average charge state ratio for $\mathrm{C}_{n}^{+}(n=5,8,10)$ ions traversing an amorphous carbon foil, as a function of the foil thickness, when the projectile energy is $2 \mathrm{MeV}$ /atom; symbols are the experimental data [5]; the solid lines are our calculations, for which we have also taken $\left\langle N_{j}\right\rangle$, instead of $\left\langle N_{j}^{*}\right\rangle$, in eq. (4). As fig. 2 shows, the vicinage effects in the charge state always increase with the molecular size. Paradoxically, our calculations (and the experimental data [5]) state that $\mathcal{R}$ for a triangular $\mathrm{C}_{3}^{+}$ion is closer to one than for a linear $\mathrm{C}_{5}^{+}$ion, although the average number of closest neighbours is larger in the former case. This phenomenon could be attributed to the asymmetrical screening of the atomic ions at the exit of the surface.

In all the cases depicted in figs. 1 and 2, the vicinage effects in the charge state decrease the thicker is the foil, tending to unity as the target thickness increases. This is a general trend that can be explained because the vicinage effects are smaller the larger the internuclear distances $R_{k j}$, which grow with the time taken to cross the foil. Moreover, the average charge 
state ratio is always smaller than unity, i.e., $\left\langle N_{j}\right\rangle\left\langle\left\langle N_{j}^{*}\right\rangle\right.$, because the presence of $(n-1)$ neighbours close to an atomic ion increases its effective nuclear charge (by increasing the magnitude of the attractive potential), which leads to a larger number of bound electrons.

After this work was finished we were aware of a paper by Mišković et al. [20] that discussed the same problem as ours and obtained similar results, but using a statistical model of the molecular structures instead of the proper geometries. These authors describe the molecule as a spherical ball, which implies a homogeneous distribution of the molecular constituents [21]. This model may be applicable to large clusters but is not useful for small molecules, as stated in ref. [22]; however, the molecules involved in this discussion are too small and with a geometry that does not warrant the validity of the spherical ball model. In addition, Mišković et al. [20] suppose that the Coulomb explosion is inhibited by the wake forces, and that the multiple nuclear scattering is the dominant process in the determination of the temporal evolution of the molecular structure. However, we have checked this assumption through a computer simulation (as suggested in ref. [20]), finding that the Coulomb explosion plays a more important role than the multiple nuclear scattering in the evolution of the molecular structure.

The surprising agreement of the results obtained by Mišković et al. [20] and the experimental data [5] could be attributed to the procedure used to determine one of the main parameters of their spherical ball model. This parameter is the exclusion distance, $d_{\mathrm{ex}}$ (i.e., the minimum separation between two atomic ions of the molecule), which is obtained in such a manner that it provides a satisfactory fitting with experimental data [5]. Moreover, the value of $d_{\mathrm{ex}}$, instead of being a geometrical characteristic of the molecule, strongly depends on the interaction potential employed to describe the interaction between the molecular contituents; this leads, for instance, to unphysical values of $d_{\mathrm{ex}}$ for a pure Coulomb interaction. Therefore, this strong unphysical dependence casts serious doubts on the validity in which the exclusion distance, $d_{\mathrm{ex}}$, is determined.

Our model describes properly the molecular structure, which clearly differs from a homogeneus sphere, and does not include any fitting parameter. We have not taken into account nuclear scattering because it is not very important for small thickness, where the vicinage effects are larger. For the larger thicknesses, where the nuclear scattering may be significant, the fragments are far away and the vicinage effects are small.

In conclusion, we have proposed a method to evaluate the average charge states of dissociated molecular ions traversing a foil. Our calculations show that it is relevant to consider the asymmetrical transient screening effects of the solid-vacuum interface as the molecular contituents leave the foil.

We have applied our model to the case of $\mathrm{N}_{2}^{+}$and $\mathrm{C}_{n}^{+}(n=3-10)$ molecular ions traversing thin carbon foils, obtaining in all cases a satisfactory agreement with the experimental charge state ratios $[3,5]$. The dependence between the charge state and the molecular size was satisfactorily well described for all the molecular ions studied here. Application of this model to heteronuclear molecular ions should be also straightforward.

The effects in the average charge state considered here should also have a direct influence on the well-known vicinage effects in the energy loss of swift molecular ions [23,24] due to modifications in the magnitude of both the interference and the self-stopping terms determining the energy loss of a cluster of ions [7].

We acknowledge financial support from the Spanish Ministerio de Ciencia y Tecnología (project BFM2000-1050-C02-01) and the Argentinian FONCYT (project PICT 0303579). SHA thanks the Spanish MEC for a research grant during his stay at the Centro Atómico Bariloche (Argentina). 


\section{REFERENCES}

[1] Gaillard M. J., Poizat J. C. and Remillieux J., Phys. Rev. Lett., 41 (1978) 159.

[2] Cue N., de Castro-Faria N. V., Gaillard M. J., Poizat J.-C., Remillieux J., Gemmell D. S. and Plesser I., Phys. Rev. Lett., 45 (1980) 613.

[3] Maor D., Cooney P. J., Faibis A., Kanter E. P., Koenig W. and Zabransky B. J., Phys. Rev. A, 32 (1985) 105.

[4] Steuer M. F. and Ritchie R. H., Nucl. Instrum. Methods Phys. Res. B, 40/41 (1989) 372.

[5] Brunelle A., Della-Negra S., Depauw J., Jacquet D., Le Y. Beyec and Pautrat M., Phys. Rev. A, 59 (1999) 4456.

6] Jensen J. and Sigmund P., Phys. Rev. A, 61 (2000) 032903.

[7] Arista N. R., Nucl. Instrum. Methods Phys. Res. B, 164-165 (2000) 108.

[8] Brandt W., in Atomic Collisions in Solids, edited by S. Datz, B. R. Appleton and C. D. MoAK, Vol. 1 (Plenum, New York) 1975. p. 261.

[9] Sigmund P., Bitensky I. S. and Jensen J., Nucl. Instrum. Methods Phys. Res. B, 112 (1996) 1.

[10] Ben-Hamu D., Baer A., Feldman H., Levin J., Heber O., Amitay Z., Vager Z. and Zajfman D., Phys. Rev. A, 15 (1997) 4786.

[11] Brandt W. and Kitagawa M., Phys. Rev. B, 25 (1982) 5631.

[12] Ziegler J. F., Biersack J. P. and Littmark U., The stopping and range of ions in solids, in The Stopping and Ranges of Ions in Solids, Vol. 1 (Pergamon, New York) 1985.

[13] Denton C., Pérez-Pérez F. J., Abril I., Garcia-Molina R. and Arista N. R., Europhys. Lett., 35 (1996) 499.

[14] N. Barberán and Pí M., Phys. Rev. B, 51 (1995) 7329.

[15] Gemmell D. S., Nucl. Instrum. Methods, 194 (1982) 255.

[16] Faibis A., Kanter E. P., Tack L. M., Bakke E. and Zabransky B. J., J. Phys. Chem., 91 (1987) 6445.

[17] Martin J. M. L., François J. P. and Gijbels R., J. Chem. Phys., 93 (1990) 5037.

[18] Watts J. D., Staton J. F., Gauss J. and Bartlett R. J., J. Chem. Phys., 94 (1990) 4320.

[19] Weltner W. J. and Von Zee R. J., Chem. Rev., 89 (1989) 1713.

[20] Mišković Z. L., Davison S. G., Goodman F. O., Liu W.-K. and Wang Y.-N., Phys. Rev. $A, 61(2000) 062901$.

[21] Mišković Z. L., Liu W.-K. and Wang Y.-N., Phys. Rev. A, 57 (1998) 362.

[22] Vicanek M., Abril I., Arista N. R. and Gras-Marti A., Phys. Rev. A, 46 (1992) 5745.

[23] Narumi K., Nakajima K., Kimura K., Mannami M., Saitoh Y., Yamamoto S., Aoki Y. and Naramoto H., Nucl. Instrum. Methods Phys. Res. B, 135 (1998) 77.

[24] Heredia-Avalos S., Garcia-Molina R. and Abril I., Nucl. Instrum. Methods Phys. Res., 164-165 (2000) 296. 